Gefässchirurgie 2009 14:348-349

DOI 10.1007/s00772-009-0739-4

Online publiziert: 16. September 2009

(c) Springer Medizin Verlag 2009

\author{
H.-H. Eckstein \\ Klinik für Gefäßchirurgie, Klinikum rechts der Isar der Technischen Universität München
}

\title{
Gefäßchirurgie 2009
}

Ein allgemeines Screening von extra-

Liebe Kolleginnen und Kollegen,

es ist mir eine außerordentliche Freude, Sie zur 25. Jahrestagung der Deutschen Gesellschaft für Gefäßchirurgie (DGG) in München begrüßen zu dürfen. Das vorliegende Kongressheft der „Gefässchirurgie“ trägt den Titel „Screening von Gefäßerkrankungen“. Dieses Schwerpunktthema wurde gewählt, da die Anzahl vermeidbarer vaskulärer Notfälle oder auch die Anzahl weit fortgeschrittener Krankheitsstadien immer noch ansteigt. Die Arbeiten dieses Heftes sollen helfen, Risikopatienten früher zu identifizieren, um rechtzeitig eine effektive Primär- oder Sekundärprävention einleiten zu können. Mein herzlicher Dank richtet sich an alle Autoren, die die vorliegenden Übersichten neben ihrer anspruchsvollen klinischen Tätigkeit in perfekter Weise erstellt haben.

Für das abdominelle Aortenaneurysma (AAA) ist die Datenlage eindeutig: Ein Ultraschallscreening ab dem 65. Lebensjahr führt zu einer signifikanten Reduktion der AAA-assoziierten Letalität und der Gesamtletalität in der gescreenten Population. Leider werden in deutschen Krankenhäusern immer noch jährlich ca. 2500 rupturierte AAAs behandelt. Aus diesem Grund hat die DGG beim DIMDI die Erstellung eines HTA- (Health-Technology-Assessment-)Berichtes beantragt, der uns helfen soll, beim Gemeinsamen Bundesausschuss (GBA) ein nationales AAAScreening-Programm erfolgreich beantragen zu können. Parallel sind wir dabei, die Fachöffentlichkeit durch entsprechende Publikationen (z. B. im Deutschen Ärzteblatt) für dieses Thema zu sensibilisieren. Auch der kommende Patiententag der DGG am 31.10.2009 wird unter dem Motto AAA-Screening stehen. kraniellen Karotisstenosen erscheint logistisch und finanziell kaum darstellbar. Dennoch bleibt es eine traurige Tatsache, dass jedes Jahr in Deutschland 20.00o30.000 karotisbedingte Schlaganfälle zu verzeichnen sind. Wir Gefäßchirurgen wissen, dass ein Großteil dieser Schlaganfälle durch eine effektivere Identifikation gefährlicher Karotisstenosen verhindert werden könnte. Das Screening älterer PAVK- und KHK-Patienten könnte ein erster Schritt in diese Richtung sein.

Die PAVK stellt eine Marker-Erkrankung für ein erhöhtes kardiovaskuläres Risiko dar. Dies hat die deutsche getABIStudie auch für klinisch noch symptomfreie Patienten eindeutig bewiesen. Es ist deshalb notwendig, diese Patienten in unseren Sprechstunden noch besser zu identifizieren und dann einer internistischkardiologischen Abklärung sowie einer optimalen medikamentösen und nichtmedikamentösen Prävention der Atherosklerose zuzuführen.

Ein weiterer Beitrag dieses Heftes berichtet von der Strategiesitzung der DGG im Juli in München. Die Informationen zu aktuellen Entwicklungen innerhalb der DGG sollen die Grundlage für weitergehende konstruktive Diskussionen darstellen. In diesem Zusammenhang bitte ich Sie sehr, die Mitgliederversammlung der DGG am Freitag, den 16. Oktober, zu besuchen, um Beschlüsse und Diskussionen innerhalb der DGG auf ein möglichst breites Fundament stellen zu können.

Unter dem Leitthema GEFÄSSCHIRURGIE 2009 - MULTIMODALE GEFÄSSMEDIZIN erwartet Sie auf dem 25. DGG-Jahreskongress die gesamte Bandbreite der heutigen Gefäßchirurgie/ Gefäßmedizin. Neben dem wissenschaft- lichen Hauptprogramm wird eine Vielzahl von Weiterbildungssymposien und Workshops angeboten. Es sollte für jeden etwas Interessantes dabei sein!

Ich wünsche Ihnen im Namen der Schriftleitung und des Herausgeberteams der "Gefässchirurgie" eine interessante Lektüre und freue mich auf ein zahlreiches Wiedersehen in München. Mit den besten Grüßen Ihr

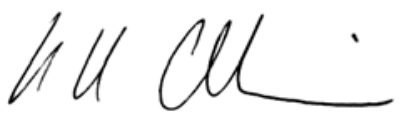

Prof. Dr. Hans-Henning Eckstein Präsident der Deutschen Gesellschaft für Gefäßchirurgie 2009/2010

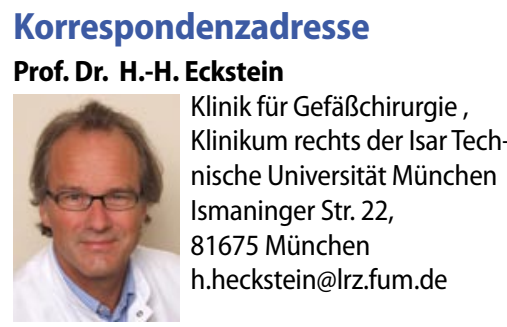


Hier steht eine Anzeige.

算 Springer 